\title{
Haptoglobin Genotype and Risk Markers of Cardiovascular Disease in Patients with Chronic Kidney Disease
}

\author{
Charlotte Strandhave, ${ }^{1,2}$ My Svensson, ${ }^{3}$ Henrik Krarup, ${ }^{4}$ and Jeppe Hagstrup Christensen ${ }^{1,2}$ \\ ${ }^{1}$ Department of Nephrology, Aalborg Hospital, Aarhus University Hospital, Moelleparkvej 4, 9000 Aalborg, Denmark \\ ${ }^{2}$ Center for Cardiovascular Research, Aalborg Hospital, Aarhus University Hospital, Aalborg, Denmark \\ ${ }^{3}$ Department of Nephrology, Aarhus University Hospital, 8200 Aarhus N, Denmark \\ ${ }^{4}$ Department of Clinical Biochemistry, Aalborg Hospital, Aarhus University Hospital, Aalborg, Denmark
}

Correspondence should be addressed to Charlotte Strandhave; charlotte.strandhave@rn.dk

Received 3 December 2012; Revised 19 January 2013; Accepted 3 February 2013

Academic Editor: Alejandro Martín-Malo

Copyright (c) 2013 Charlotte Strandhave et al. This is an open access article distributed under the Creative Commons Attribution License, which permits unrestricted use, distribution, and reproduction in any medium, provided the original work is properly cited.

Sudden cardiac death and atherosclerosis have a major impact on cardiovascular mortality in chronic kidney disease (CKD). Inflammation with elevated high-sensitive C-reactive protein (hs-CRP) is involved in both sudden cardiac death and atherosclerosis, and decreased heart rate variability (HRV) is a predictor of both sudden cardiac death and atherosclerosis. Haptoglobin (Hp) is characterised by three genotypes (1-1,2-1, and 2-2) with different antioxidant abilities. The aim was to examine whether HRV and hs-CRP were associated with Hp genotype in CKD patients. Fifty-six patients with CKD stage $2-5$ were included. Hp genotype was determined by high-performance liquid chromatography. HRV was analysed from the 24 h Holter recordings. Hs-CRP was measured using an immunoturbidimetric assay. The results show that the HRV indices SDNN and SDANN were significantly lower in the Hp 2-2 patients ( $P=0.02$ and 0.04, resp.). In an adjusted linear regression model, Hp 2-2 was associated with both SDNN $(P=0.005)$ and SDANN $(P=0.01)$. Hs-CRP was higher in the Hp 2-2 patients $(P=0.002)$. In an adjusted linear regression model, the association between Hp 2-2 and hs-CRP remained significant $(P=0.003)$. In conclusion, a negative association was observed between Hp 2-2 and HRV, and Hp 2-2 was positively associated with hs-CRP in CKD patients.

\section{Introduction}

Cardiovascular disease (CVD) including sudden cardiac death (SCD) [1] is a major cause of mortality in chronic kidney disease (CKD) [2]. Apart from left ventricular dysfunction [1], unique factors such as autonomic imbalance and inflammation contribute to the high incidence of SCD in CKD [3]. Traditional risk factors for atherosclerosis [4], such as hypertension and dyslipidaemia, can to some extent explain the increased risk of atherosclerosis in CKD [5]. However, nontraditional risk factors like oxidative stress and inflammation may also be of importance [6].

Heart rate variability (HRV) is a reliable, noninvasive measurement of autonomic tone of the heart [7]. A reduction in HRV reflects an autonomic imbalance towards decreased vagal tone and subsequent sympathetic overactivity [3].
Decreased HRV is a predictor of SCD in patients with CKD [8]. Furthermore, attenuated HRV is associated with atherosclerosis in CKD [9].

Chronic, low-grade inflammation is involved in the development of both SCD [10] and atherosclerosis [11]. The most extensively examined marker of inflammation is highsensitive C-reactive protein (hs-CRP), which is a predictor of cardiovascular mortality in different populations [12, 13] including patients with CKD [14]. Hs-CRP may even be a risk marker for SCD $[10,15]$.

Haptoglobin (Hp) is an abundant acute-phase protein as well as an innate antioxidant involved in the scavenging of free haemoglobin [16]. Thus, Hp prevents iron-mediated formation of free reactive oxygen species known to initiate oxidative stress [17]. In humans, $\mathrm{Hp}$ is characterised by molecular heterogeneity with three common genotypes: 1-1, 
2-2, and 2-1 [18]. The antioxidant ability of Hp is genotype dependent with Hp 2-2 being the weakest antioxidant [19]. There is evidence establishing Hp 2-2 as a predictor of CVD in diabetes mellitus [20,21]. However, results are inconsistent regarding $\mathrm{Hp}$ genotypes and cardiovascular mortality in patients with CKD $[22,23]$. A study in patients with ischemic heart disease (IHD) revealed a positive association between low HRV and Hp 2-2 [24].

The aim of the present study was to determine whether Hp genotype was associated with the cardiovascular risk markers HRV and hs-CRP in patients with CKD.

\section{Material and Methods}

2.1. Study Population. Sixty-four patients from the Outpatient Clinic at the Department of Nephrology, Aalborg Hospital, Denmark, were included. Inclusion criterion was CKD stage 2-5. Exclusion criteria were the start of dialysis within three months, nephrotic syndrome, malignancy, uncontrolled hypertension, or a previous renal transplantation. Patients were also excluded if they had an ongoing infection, a myocardial infarction during the last six months, or supraventricular arrhythmia.

Six patients with diabetes were excluded. Two patients were excluded due to technical problems. Thus, the final analysis comprised 56 patients.

The causes of CKD were chronic glomerulonephritis (32\%), hypertensive nephropathy (23\%), polycystic kidney disease (13\%), and other causes including interstitial nephritis and chronic pyelonephritis (32\%).

The study was conducted in accordance with Danish Legislation and the Declaration of Helsinki and approved by Research Ethics Committee of The North Denmark Region. Signed informed consent was obtained from all participants.

2.2. Laboratory Measurements. Blood samples were drawn after an overnight fast. All routine analyses were performed according to the standard procedures in the hospital laboratory. Creatinine clearance was calculated using a $24 \mathrm{~h}$ urine collection and a blood sample for serum creatinine. Hs-CRP was measured using an ADVIA 1650 analyzer (Bayer Corp., Pittsburgh, PA, USA) and an immunoturbidimetric assay (Randox Laboratories Ltd., Antrim, UK). Detection level was $0.2 \mathrm{mg} / \mathrm{L}$. The analysis was conducted in accordance with the instruction of the manufacturer.

The Hp genotype was determined by high-performance liquid chromatography as previously described [25], using a Superdex $200 \mathrm{HR}$ 10/30 column for chromatographic separation (Amersham Pharmacia Biotech AB, Birkerød, Denmark). Phosphate-buffered saline $(50 \mathrm{mmol} / \mathrm{L}$ sodium phosphate, $150 \mathrm{mmol} / \mathrm{L}$ sodium chloride, and $0.01 \%$ sodium azide; $\mathrm{pH} 7.3$ ) was used as the mobile phase. Flow rate was $1 \mathrm{~mL} / \mathrm{min}$ ( $76.4 \mathrm{~cm} /$ hour). Ten $\mu \mathrm{L}$ was used for analysis. Hp genotype was determined from the curve shape and relative retention time of the chromatograms.

2.3. Heart Rate Variability Measurement. A 24 h Holter recording was obtained on a flash card using a 3-channel digital monitor DL-700 (Diagnostic Monitoring, Santa Ana, CA, USA), and time domain variables were analysed using commercially available software (Diagnostic Monitoring, Santa Ana, CA, USA). If more than $20 \%$ of QRS complexes were abnormal or of poor technical quality, the analysis was excluded in the overall HRV analysis. The time domain variables were identified as follows: (1) mean of all normalto-normal RR intervals, (2) standard deviation (SD) of all normal-to-normal RR intervals (SDNN), (3) mean of the SDs of all normal-to-normal RR intervals (SDNNindex), (4) SD of the mean normal-to-normal RR intervals for each 5-minute period (SDANN), (5) percentage of successive RR interval differences $>50 \mathrm{~ms}$ ( $\mathrm{pNN50}$ ), and (6) square root of the mean of the sum of squares of differences between adjacent RR intervals (RMSSD). Heart rate was calculated as the inverse value of the $R R$ variable.

2.4. Blood Pressure Measurement. Blood pressure was obtained with a $24 \mathrm{~h}$ ambulatory BP device, TM 2412 (A\&D Co. Ltd., Tokyo, Japan). For analysis, average values during daytime for systolic and diastolic blood pressure were calculated.

2.5. Statistical Analysis. The statistical analysis was performed using Stata software, version 10 (StataCorp LP, TX, USA). Continuous data were expressed as mean values \pm SD if normal distributed. Nonnormal distributed data were log transformed and expressed as geometric mean and ranges. The Hp alleles were tested for Hardy-Weinberg equilibrium using an asymptotic Hardy-Weinberg equilibrium test.

For the comparison of SDNN, SDANN, and hs-CRP, respectively, between the three common Hp genotypes, crude one-way analysis of variance was performed.

Data was divided into two groups according to the presence of the $\mathrm{Hp}$ allele 1: one group including $\mathrm{Hp}$ 1-1 and Hp 2-1 patients and the remaining group consisting of Hp 2-2 patients. For comparisons of groups, Student's $t$-test was used for continuous variables and Fisher's exact test for categorical variables. Linear regression was used to analyse the effect of Hp 2-2 compared to Hp 1-1 and Hp 2-1 on SDNN, SDANN, and hs-CRP, respectively. The analyses were performed with the Hp group as the independent variable and SDNN, SDANN, and hs-CRP, respectively, as the dependent variables. Adjustments for potential confounders described in previous studies (age, gender, heart rate, beta-blocker treatment, and creatinine clearance for HRV indices and age, gender, body mass index, and smoking status for hs-CRP) [26-28] were performed. Regression coefficients represented a difference between the two Hp genotype groups. A $P$ value $<0.05$ was considered statistically significant.

\section{Results}

The frequencies of the three common genotypes were Hp 1-1 21\%, Hp 2-1 50\%, and Hp 2-2 29\%, and the Hp allele frequencies (Hp allele 10.457 and Hp allele 20.544 ) were in Hardy-Weinberg equilibrium $\left(\chi^{2}=2.07\right.$ and $\left.P=0.1\right)$. The genotype frequencies in the cohort were similar to those reported previously in northwestern European countries 


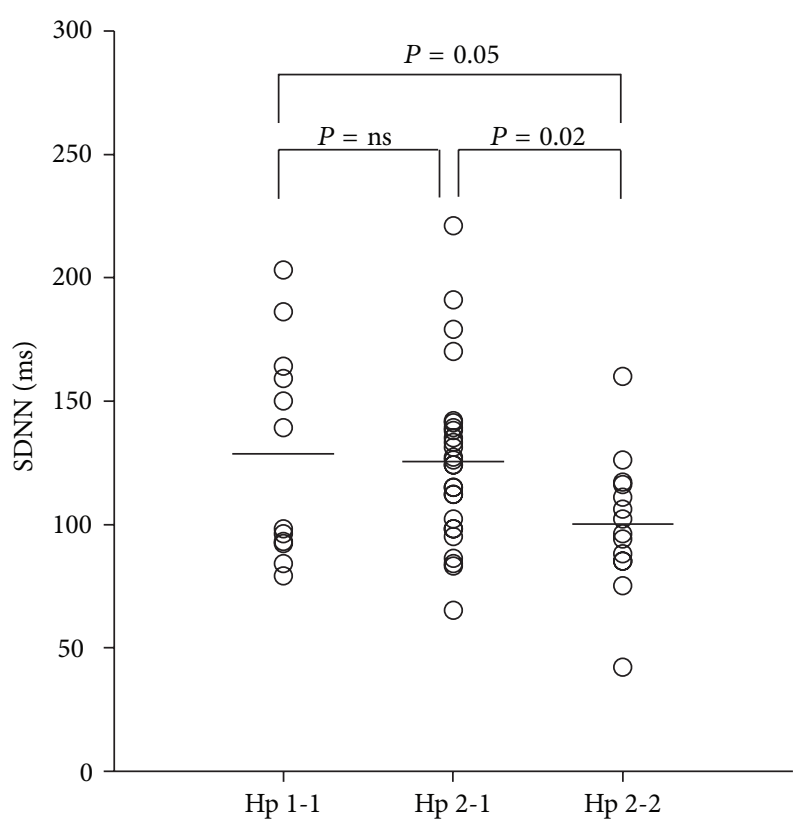

(a)

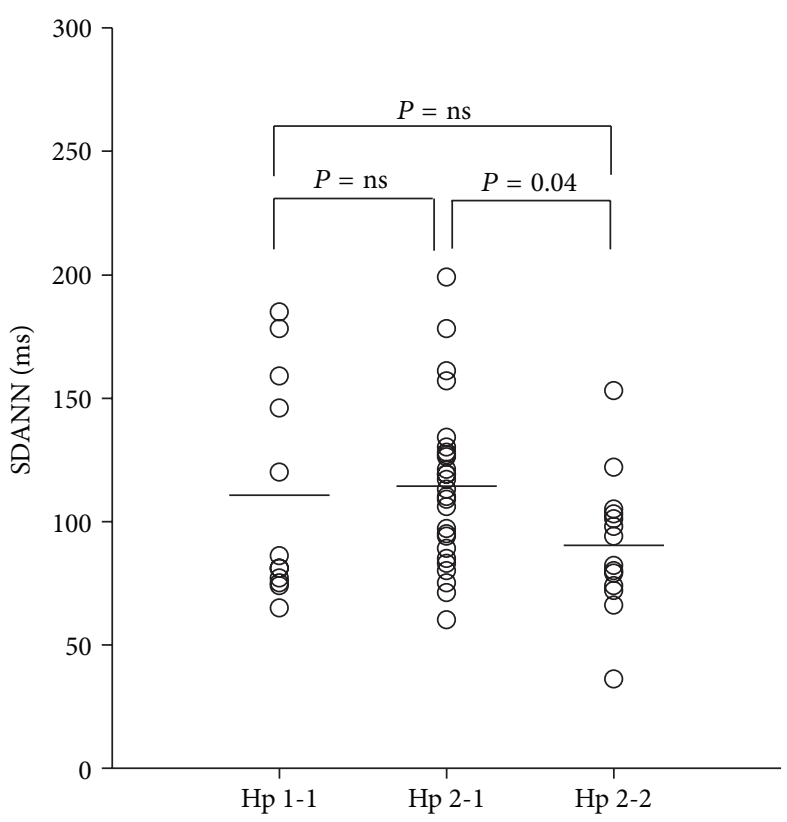

(b)

FIGURE 1: Dot plots showing (a) SDNN (ms) and (b) SDANN (ms) in haptoglobin genotypes 1-1, 2-1, and 2-2, respectively. The lines represent mean values. Abbreviations Hp: haptoglobin.

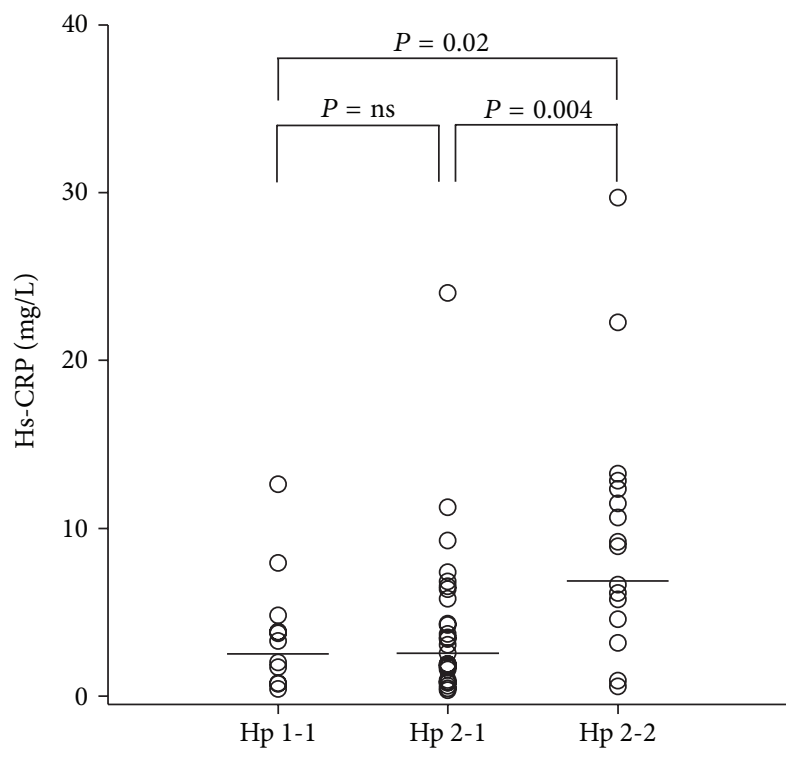

FIgURE 2: Dot plot showing high-sensitive C-reactive protein $(\mathrm{mg} / \mathrm{L})$ in haptoglobin genotypes 1-1, 2-1, and 2-2, respectively. The lines represent mean values. Abbreviations: Hp: haptoglobin and hsCRP: high-sensitive C-reactive protein.

$\left(\chi^{2}=3.42\right.$ and $\left.P=0.2\right)[29]$ and similar to those reported previously in a Danish population $\left(\chi^{2}=3.40\right.$ and $\left.P=0.2\right)$ [30].

Figure 1 depicts mean SDNN and SDANN, and Figure 2 mean hs-CRP in the three common Hp genotypes. No differences were demonstrated between Hp 1-1 and Hp 2-1 regarding SDNN, SDANN, and hs-CRP, respectively.
However, significant differences were observed between $\mathrm{Hp}$ 1-1 and Hp 2-2 and between Hp 2-1 and Hp 2-2 for both SDNN and hs-CRP. However, for SDANN, a significant difference was demonstrated only between Hp 2-1 and Hp 2-2.

Table 1 shows clinical characteristics in the Hp genotypes divided into two groups according to the presence of $\mathrm{Hp}$ allele 1 . The two groups were generally comparable, although there were fewer women in the Hp 2-2 group.

SDNN and SDANN were significantly lower in the Hp 2-2 patients compared to the Hp 1-1 and Hp 2-1 patients (Table 1). The other time domain indices RR, SDNNindex, pNN50, and RMSSD showed a similar trend although not statistically significant. Multivariate linear regression analyses of $\mathrm{Hp} 2$ 2 and SDNN and SDANN, respectively, were performed adjusting for previously described confounders [26]. Hp 22 was negatively and independently associated with both SDNN and SDANN (Table 2). Apart from Hp 2-2, the strongest determinants of SDNN in the model were age $(P<0.001)$ and heart rate $(P=0.04)$, and the strongest determinants of SDANN were age $(P<0.001)$, heart rate $(P=0.05)$, and beta-blocker treatment $(P=0.04)$. After adjusting for age, gender, heart rate, creatinine clearance, and beta-blocker treatment, Hp 2-2 was still significantly associated with both SDNN and SDANN.

Levels of hs-CRP were significantly higher in the Hp 2-2 patients compared to the Hp 1-1 and Hp 2-1 patients (Table 1). In the multiple linear regression analysis of hs-CRP and Hp 2-2 (Table 2), adjustments were made for confounders known from previous studies $[27,28]$. The association between $\mathrm{Hp}$ genotype and hs-CRP remained significant although attenuated. Only Hp 2-2 revealed a positive and significant association with hs-CRP in the model. 
TABLE 1: Clinical characteristics of patients with haptoglobin genotype 2-2 compared to patients with haptoglobin genotypes 1-1 and 2-1.

\begin{tabular}{|c|c|c|c|}
\hline & $\begin{array}{l}\text { Haptoglobin genotypes } 1-1 \text { and 2-1 } \\
\qquad(n=40)\end{array}$ & $\begin{array}{l}\text { Haptoglobin genotype } 2-2 \\
\qquad(n=16)\end{array}$ & $P$ \\
\hline Age (y) & $60 \pm 11$ & $56 \pm 13$ & ns \\
\hline Women $(n)$ & $18(45 \%)$ & $2(13 \%)$ & 0.03 \\
\hline Smoking $(n)$ & $14(35 \%)$ & $4(25 \%)$ & ns \\
\hline Body mass index $\left(\mathrm{kg} / \mathrm{m}^{2}\right)$ & $28 \pm 5$ & $30 \pm 5$ & ns \\
\hline Beta-blocker treatment $(n)$ & $16(42 \%)$ & $7(44 \%)$ & ns \\
\hline \multicolumn{4}{|l|}{ Haemodynamic parameters } \\
\hline Systolic BP (mmHg) & $142 \pm 18$ & $140 \pm 18$ & ns \\
\hline Diastolic BP (mmHg) & $78 \pm 10$ & $80 \pm 9$ & ns \\
\hline Heart rate (beats per min) & $72 \pm 10$ & $77 \pm 11$ & ns \\
\hline \multicolumn{4}{|l|}{ Laboratory parameters } \\
\hline Creatinine clearance $(\mathrm{mL} / \mathrm{min})$ & $35 \pm 18$ & $45 \pm 20$ & ns \\
\hline Haemoglobin (mmol/L) & $7.6 \pm 1$ & $8.0 \pm 1$ & ns \\
\hline Hs-CRP (mg/L) & $2.5(0.3 ; 24.0)$ & $6.9(0.6 ; 29.7)$ & 0.002 \\
\hline \multicolumn{4}{|l|}{ HRV indices } \\
\hline $\mathrm{RR}$ (ms) & $853 \pm 124$ & $790 \pm 113$ & ns \\
\hline SDNN (ms) & $126 \pm 37$ & $100 \pm 27$ & 0.02 \\
\hline SDNNindex (ms) & $45(39 ; 51)$ & $39(33 ; 45)$ & ns \\
\hline SDANN (ms) & $113 \pm 36$ & $90 \pm 28$ & 0.04 \\
\hline pNN50 (\%) & $5.5(3.5 ; 8.8)$ & $3.4(1.6 ; 7.1)$ & ns \\
\hline RMSSD (ms) & $24(20 ; 30)$ & $22(17 ; 28)$ & ns \\
\hline
\end{tabular}

Values are mean \pm SD or number of patients (\%), except from high-sensitive C-reactive protein and heart rate variability indices SDNNindex, pNN50, and RMSSD which are given in geometric mean and ranges.

Abbreviations: BP: blood pressure, hs-CRP: high-sensitive C-reactive protein, and HRV: heart rate variability.

\section{Discussion}

The present study demonstrated a close association between Hp genotype and the cardiovascular risk markers HRV and hs-CRP in patients with CKD. Thus, patients with Hp 2-2 had significantly lower HRV and higher levels of hs-CRP.

\subsection{Haptoglobin Genotype and Risk of Sudden Cardiac Death.} Kanbay et al. establishes an SCD as one of the most single important causes of death in patients with CKD [3]. Welldescribed risk factors of SCD in the general population also apply to patients with $\mathrm{CKD}$ [3]. However, several unique factors such as autonomic imbalance and inflammation are recognized [15]. Schwartz has established the importance of cardiac autonomic control in the pathogenesis of SCD [31]. HRV is being increasingly used as a valid and easily obtainable prognostic marker of autonomic alterations [7]. As reviewed by Ranpuria et al., HRV is a promising surrogate marker for SCD in patients with CKD and a potential modifiable outcome measure [32].

In haemodialysis patients, Oikawa et al. found decreased SDNN to be a predictor of all-cause and cardiovascular mortality [8]. Recently, Chandra et al. studied HRV in patients with CKD stage 3-5 demonstrating that lower HRV was independently associated with higher risk of CVD and mortality [33]. Christensen et al. has previously addressed the issue of a possible association between HRV and Hp genotype [24]. In patients with IHD, a significant association between time domain variable RMSSD and Hp 2-2
TABLE 2: Differences in SDNN, SDANN, and high-sensitive Creactive protein between patients with haptoglobin genotype 2-2 compared to patients with haptoglobin genotypes 1-1 and 2-1.

\begin{tabular}{|c|c|c|}
\hline & $\begin{array}{c}\text { Difference between } \\
\text { haptoglobin 2-2 and } \\
\text { haptoglobins 1-1 and 2-1 } \\
(95 \% \mathrm{CI})\end{array}$ & $P$ value \\
\hline \multicolumn{3}{|l|}{ SDNN } \\
\hline Crude* & $-26.2(-48 ;-4)$ & 0.02 \\
\hline Adjusted ${ }^{* *}$ & $-31.7(-53 ;-10)$ & 0.005 \\
\hline \multicolumn{3}{|l|}{ SDANN } \\
\hline Crude* & $-22.7(-44 ;-1)$ & 0.04 \\
\hline Adjusted $^{* *}$ & $-27.4(-48 ;-7)$ & 0.01 \\
\hline \multicolumn{3}{|c|}{$\begin{array}{l}\text { High-sensitive C-reactive } \\
\text { protein }\end{array}$} \\
\hline Crude* & $2.7(2 ; 5)$ & 0.002 \\
\hline Adjusted ${ }^{* * *}$ & $2.7(1 ; 5)$ & 0.003 \\
\hline \multicolumn{3}{|c|}{$\begin{array}{l}{ }^{\dagger} \text { Difference presented as regression coefficient and } 95 \% \text { CI. Haptoglobin } \\
\text { genotypes } 1-1 \text { and } 2-1 \text { are the reference level. } \\
{ }^{*} \text { Univariate linear regression analysis. } \\
{ }^{* *} \text { Multivariate linear regression analysis adjusted for age, gender, heart rate, } \\
\text { creatinine clearance, and beta-blocker treatment. } \\
{ }^{* * *} \text { Multivariate linear regression analysis adjusted for age, gender, body } \\
\text { mass index, and smoking status. }\end{array}$} \\
\hline
\end{tabular}

was shown. RMSSD primarily reflects the short-term vagal modulation of the sinoatrial node. The present study did not 
show an association between RMSSD and Hp 2-2. However, a highly significant association between $\mathrm{Hp}$ 2-2 and SDNN and SDANN, primarily reflecting circadian rhythm and longterm fluctuations, was demonstrated.

A relationship between the autonomic nervous system and inflammation has been established showing increase in vagal tone as a counter-response to cytokine synthesis [34]. Tracey [35] first described this cholinergic antiinflammatory pathway as a plausible, molecular mechanism for the association between HRV and hs-CRP. In a cohort of patients with CKD, Psychari et al. demonstrated a negative association between inflammatory marker interleukin- 6 and SDNN and SDANN, respectively [36]. Furthermore, in a large cohort of end-stage renal disease patients, Parekh et al. [15] found a positive association between hs-CRP and SCD, independently of other known cardiovascular risk factors. Direct effects on the vagal conduction system and resulting aggravation of sympathetic tone may contribute to the increased risk of SCD [15]. Being the weaker antioxidant of the three common Hp genotypes, Hp 2-2 may be unable to hinder oxidative damage to the autonomic nervous system, especially in patients with CKD exposed to a prooxidative milieu due to uremic toxins. Thereby, $\mathrm{Hp} 2-2$ may potentially contribute to a decreased HRV.

4.2. Haptoglobin Genotype and Risk of Atherosclerosis. Studies have established Hp 2-2 as a potential risk predictor of CVD in patients without CKD. Chapelle et al. [37] showed that more extensive myocardial infarction occurred in $\mathrm{Hp} 2$ 2 patients. Delanghe et al. demonstrated that Hp 2-2 patient undergoing coronary bypass surgery needed more grafts and had shorter graft survival time [38]. On the contrary, in a nested control study de Bacquer et al. showed that Hp 11 patients had a higher risk of IHD compared to Hp 2-2 and Hp 2-1 patients [39]. Studies on CVD and Hp genotype in diabetes show more consistent results, both in human studies [21] and recently in a mouse model [40]. Levy et al. demonstrated in the Strong Heart study [20] that Hp 2-2 is a major predictor of CVD in patients with diabetes. Yet, in a cross-sectional study of the Framingham Offspring Cohort [41], Levy et al. found that Hp 2-2 and Hp 2-1 patients with diabetes had decreased CVD prevalence.

A possible relation between hs-CRP and Hp 2-2 has been examined in several studies. In patients with peripheral occlusive disease, Delanghe et al. [42] found a higher, yet statistically insignificant, hs-CRP in Hp 2-2 patients compared to Hp 1-1 and Hp 2-1 patient. Likewise, Braeckman et al. [43] found a higher, but also statistically insignificant, hs-CRP in apparently healthy $\mathrm{Hp}$ 2-2 individuals compared to Hp 1-1 and Hp 2-1 individuals. However, Delanghe et al. demonstrated in patients with essential hypertension higher hs-CRP in $\mathrm{Hp}$ 2-2 patients [44]. Recently, Mohieldein et al. found no association between Hp and markers of inflammation in diabetes [45]. Unfortunately, renal function was evaluated in none of these studies.

The present study establishes a potential role for Hp 22 as a risk predictor of CVD in patients with CKD through an association with hs-CRP. Numerous studies have documented hs-CRP to be a valid and well-documented marker of inflammation [11] and hence a predictor of atherosclerosis and cardiovascular mortality in patients with IHD [13] as well as in patients with CKD, as recently demonstrated by Bazeley et al. [14].

In patients with $\mathrm{CKD}$, results on $\mathrm{Hp}$ genotype and CVD are conflicting. Pernod et al. found a prevalence of $\mathrm{Hp} \mathrm{2-2}$ twice as high in end-stage renal disease patients with high CVD risk, defined as prior cardiovascular event, diabetes, or dyslipidemia, compared to end-stage renal disease patients with low CVD risk [46]. In a group of end-stage renal disease patients with diabetes, Burbea et al. [22] found a survival advantage in Hp 1-1 patients above 60 years, whereas patients below 60 years demonstrated a better survival when having Hp 2-2. However, Pernod et al. found no association between Hp genotype and cardiovascular mortality in patients with end-stage renal disease [23].

Several mechanisms for the differences in antioxidant capability between Hp genotypes have been proposed. Lipid oxidation plays a crucial role in the initiation of atherosclerosis [47], and the first step in lipid oxidation is mediated by redox-active metals such as iron. Brouwers et al. demonstrated that Hp 2-2 is associated with high circulating levels of oxidised low-density lipoprotein thus posing a potential proatherogenic pathway for Hp 2-2 [48]. In an animal model of knock-in Hp 2-2 mice, Levy et al. demonstrated that Hp contributes to atherosclerotic plaque modulation by increasing the local inflammation. Hp 2-2 plaques accumulate more macrophages and have decreased levels of antiinflammatory cytokines [49]. Furthermore, Hp 2-2 has been associated with diminished nitric oxide bioavailability $[50,51]$, a crucial step in endothelial dysfunction and hence initiation of atherosclerosis.

4.3. Limitations. Our study has some limitations. The cohort is relatively small and consists of patients with four stages of CKD. Furthermore, the underlying renal disease pattern is divers and comprises diseases with both high and low levels of inflammation. The design of the study is cross-sectional potentiating a risk of bias, and the causal inference cannot be established.

\section{Conclusion}

In conclusion, in patients with $\mathrm{CKD}$, a negative association was observed between Hp 2-2 and HRV. Furthermore, Hp 22 was positively associated with hs-CRP. Thus, Hp 2-2 may indicate a poor outcome in patients with $\mathrm{CKD}$, and one may speculate that Hp 2-2 can help predict cardiovascular risk in patients with CKD as previously demonstrated in patients with diabetes.

\section{Conflict of Interests}

The authors report no conflicts of interests. The authors alone are responsible for the content and writing of the paper. 


\section{Acknowledgments}

The authors thank the laboratory technicians Inge Valbak Aardestrup, Charlotte Mose Skov, and Anne BentzenPetersen for their meticulous work. Also, they express their gratitude to the patients participating in this study.

\section{References}

[1] C. A. Herzog, J. M. Mangrum, and R. Passman, "Sudden cardiac death and dialysis patients," Seminars in Dialysis, vol. 21, no. 4, pp. 300-307, 2008.

[2] R. N. Foley, P. S. Parfrey, and M. J. Sarnak, "Clinical epidemiology of cardiovascular disease in chronic renal disease," American Journal of Kidney Diseases, vol. 32, no. 5, supplement 3, pp. S112-S119, 1998.

[3] M. Kanbay, B. Afsar, D. Goldsmith, and A. Covic, "Sudden death in hemodialysis: an update," Blood Purification, vol. 30, no. 2, pp. 135-145, 2010.

[4] A. K. Cheung, M. J. Sarnak, G. Yan et al., "Atherosclerotic cardiovascular disease risks in chronic hemodialysis patients," Kidney International, vol. 58, no. 1, pp. 353-362, 2000.

[5] M. G. Shlipak, L. F. Fried, M. Cushman et al., "Cardiovascular mortality risk in chronic kidney disease: comparison of traditional and novel risk factors," Journal of the American Medical Association, vol. 293, no. 14, pp. 1737-1745, 2005.

[6] F. Locatelli, B. Canaud, K. U. Eckardt, P. Stenvinkel, C. Wanner, and C. Zoccali, "Oxidative stress in end-stage renal disease: an emerging treat to patient outcome," Nephrology Dialysis Transplantation, vol. 18, no. 7, pp. 1272-1280, 2003.

[7] European Task Force, "Heart rate variability: standards of measurement, physiological interpretation and clinical use. Task Force of the European Society of Cardiology and the North American Society of Pacing and Electrophysiology," Circulation, vol. 93, no. 5, pp. 1043-1065, 1996.

[8] K. Oikawa, R. Ishihara, T. Maeda et al., "Prognostic value of heart rate variability in patients with renal failure on hemodialysis," International Journal of Cardiology, vol. 131, no. 3, pp. 370377, 2009.

[9] J. C. Longenecker, M. Zubaid, K. V. Johny et al., "Association of low heart rate variability with atherosclerotic cardiovascular disease in hemodialysis patients," Medical Principles and Practice, vol. 18, no. 2, pp. 85-92, 2009.

[10] C. M. Albert, J. Ma, N. Rifai, M. J. Stampfer, and P. M. Ridker, "Prospective study of C-reactive protein, homocysteine, and plasma lipid levels as predictors of sudden cardiac death," Circulation, vol. 105, no. 22, pp. 2595-2599, 2002.

[11] R. Ross, "Atherosclerosis-an inflammatory disease," The New England Journal of Medicine, vol. 340, no. 2, pp. 115-126, 1999.

[12] P. M. Ridker, M. Cushman, M. J. Stampfer, R. P. Tracy, and C. H. Hennekens, "Inflammation, aspirin, and the risk of cardiovascular disease in apparently healthy men," The New England Journal of Medicine, vol. 336, no. 14, pp. 973-979, 1997.

[13] F. Haverkate, S. G. Thompson, S. D. M. Pyke, J. R. Gallimore, and M. B. Pepys, "Production of C-reactive protein and risk of coronary events in stable and unstable angina," The Lancet, vol. 349, no. 9050, pp. 462-466, 1997.

[14] J. Bazeley, B. Bieber, Y. Li et al., "C-reactive protein and prediction of 1-year mortality in prevalent hemodialysis patients," Clinical Journal of the American Society of Nephrology, vol. 6, no. 10, pp. 2452-2461, 2011.
[15] R. S. Parekh, L. C. Plantinga, W. H. L. Kao et al., "The association of sudden cardiac death with inflammation and other traditional risk factors," Kidney International, vol. 74, no. 10, pp. 1335-1342, 2008.

[16] M. R. Langlois and J. R. Delanghe, "Biological and clinical significance of haptoglobin polymorphism in humans," Clinical Chemistry, vol. 42, no. 10, pp. 1589-1600, 1996.

[17] P. M. Guéye, N. Glasser, G. Férard, and J. M. Lessinger, "Influence of human haptoglobin polymorphism on oxidative stress induced by free hemoglobin on red blood cells," Clinical Chemistry and Laboratory Medicine, vol. 44, no. 5, pp. 542-547, 2006.

[18] O. Smithies, "An improved procedure for starch-gel electrophoresis: further variations in the serum proteins of normal individuals," The Biochemical Journal, vol. 71, no. 3, pp. 585-587, 1959.

[19] S. M. Sadrzadeh and J. Bozorgmehr, "Haptoglobin phenotypes in health and disorders," American Journal of Clinical Pathology, vol. 121, supplement, pp. S97-S104, 2004.

[20] A. P. Levy, I. Hochberg, K. Jablonski et al., "Haptoglobin phenotype is an independent risk factor for cardiovascular disease in individuals with diabetes: the strong heart study," Journal of the American College of Cardiology, vol. 40, no. 11, pp. 1984-1990, 2002.

[21] R. Asleh and A. P. Levy, "In vivo and in vitro studies establishing haptoglobin as a major susceptibility gene for diabetic vascular disease," Vascular Health and Risk Management, vol. 1, no. 1, pp. 19-28, 2005.

[22] Z. Burbea, F. Nakhoul, R. Zoabi et al., "Haptoglobin phenotype as a predictive factor of mortality in diabetic haemodialysis patients," Annals of Clinical Biochemistry, vol. 41, no. 6, pp. 469473, 2004.

[23] G. Pernod, J. L. Bosson, D. Golshayan et al., "Phenotypic and genotypic risk factors for cardiovascular events in an incident dialysis cohort," Kidney International, vol. 69, no. 8, pp. 14241430, 2006.

[24] J. H. Christensen, H. B. Krarup, S. Riahi, E. Toft, and E. B. Schmidt, "Heart rate variability is associated with haptoglobin phenotype in patients with coronary artery disease," European Journal of Cardiovascular Prevention and Rehabilitation, vol. 12, no. 3, pp. 221-225, 2005.

[25] J. Delanghe, K. Allcock, M. Langlois, L. Claeys, and M. De Buyzere, "Fast determination of haptoglobin phenotype and calculation of hemoglobin binding capacity using high pressure gel permeation chromatography," Clinica Chimica Acta, vol. 291, no. 1, pp. 43-51, 2000.

[26] K. Tamura, H. Tsuji, T. Nishiue, I. Yajima, T. Higashi, and T. Iwasaka, "Determinants of heart rate variability in chronic hemodialysis patients," American Journal of Kidney Diseases, vol. 31, no. 4, pp. 602-606, 1998.

[27] P. Stenvinkel, C. Wanner, T. Metzger et al., "Inflammation and outcome in end-stage renal failure: does female gender constitute a survival advantage?" Kidney International, vol. 62, no. 5, pp. 1791-1798, 2002.

[28] N. Ramkumar, A. K. Cheung, L. M. Pappas, W. L. Roberts, and S. Beddhu, "Association of obesity with inflammation in chronic kidney disease: a cross-sectional study," Journal of Renal Nutrition, vol. 14, no. 4, pp. 201-207, 2004.

[29] K. Carter and M. Worwood, "Haptoglobin: a review of the major allele frequencies worldwide and their association with diseases," International Journal of Laboratory Hematology, vol. 29, no. 2, pp. 92-110, 2007. 
[30] F. Galatius-Jensen, The Haptoglobins. A Genetical Study, Dansk Videnskabs Forlag, Copenhagen, Denmark, 1960.

[31] P. J. Schwartz, "The autonomic nervous system and sudden death," European Heart Journal, vol. 19, pp. F72-F80, 1998.

[32] R. Ranpuria, M. Hall, C. T. Chan, and M. Unruh, "Heart rate variability (HRV) in kidney failure: measurement and consequences of reduced HRV," Nephrology Dialysis Transplantation, vol. 23, no. 2, pp. 444-449, 2008.

[33] P. Chandra, R. L. Sands, B. W. Gillespie et al., "Predictors of heart rate variability and its prognostic significance in chronic kidney disease," Nephrology Dialysis Transplantation, vol. 27, no. 2, pp. 700-709, 2012.

[34] J. M. Huston and K. J. Tracey, “The pulse of inflammation: heart rate variability, the cholinergic anti-inflammatory pathway and implications for therapy," Journal of Internal Medicine, vol. 269, no. 1, pp. 45-53, 2011.

[35] K. J. Tracey, "Physiology and immunology of the cholinergic antiinflammatory pathway," Journal of Clinical Investigation, vol. 117, no. 2, pp. 289-296, 2007.

[36] S. N. Psychari, L. Sinos, C. Iatrou, G. Liakos, and T. S. Apostolou, "Relations of inflammatory markers to lipid levels and autonomic tone in patients with moderate and severe chronic kidney disease and in patients under maintenance hemodialysis," Clinical Nephrology, vol. 64, no. 6, pp. 419-427, 2005.

[37] J. P. Chapelle, A. Albert, and J. P. Smeets, "Effect of the haptoglobin phenotype on the size of a myocardial infarct," The New England Journal of Medicine, vol. 307, no. 8, pp. 457-463, 1982.

[38] J. Delanghe, B. Cambier, M. Langlois et al., "Haptoglobin polymorphism, a genetic risk factor in coronary artery bypass surgery," Atherosclerosis, vol. 132, no. 2, pp. 215-219, 1997.

[39] D. De Bacquer, G. De Backer, M. Langlois, J. Delanghe, H. Kesteloot, and M. Kornitzer, "Haptoglobin polymorphism as a risk factor for coronary heart disease mortality," Atherosclerosis, vol. 157, no. 1, pp. 161-166, 2001.

[40] R. Asaf, S. Blum, A. Roguin et al., "Haptoglobin genotype is a determinant of survival and cardiac remodeling after myocardial infarction in diabetic mice," Cardiovascular Diabetology, vol. 8, article 29, 2009.

[41] A. P. Levy, M. G. Larson, D. Corey, R. Lotan, J. A. Vita, and E. J. Benjamin, "Haptoglobin phenotype and prevalent coronary heart disease in the Framingham offspring cohort," Atherosclerosis, vol. 172, no. 2, pp. 361-365, 2004.

[42] J. Delanghe, M. Langlois, D. Duprez, M. De Buyzere, and D. Clement, "Haptoglobin polymorphism and peripheral arterial occlusive disease," Atherosclerosis, vol. 145, no. 2, pp. 287-292, 1999.

[43] L. Braeckman, D. De Bacquer, J. Delanghe, L. Claeys, and G. De Backer, "Associations between haptoglobin polymorphism, lipids, lipoproteins and inflammatory variables," Atherosclerosis, vol. 143, no. 2, pp. 383-388, 1999.

[44] J. R. Delanghe, D. A. Duprez, M. L. De Buyzere et al., "Haptoglobin polymorphism and complications in established essential arterial hypertension," Journal of Hypertension, vol. 11, no. 8, pp. 861-867, 1993.

[45] A. Mohieldein, M. Alzohairy, M. Hasan, and A. A. Khan, "Inflammatory markers and haptoglobin polymorphism in Saudi with non-insulin-dependent diabetes mellitus," Global Journal of Health Science, vol. 5, no. 1, pp. 135-142, 2012.

[46] G. Pernod, J. L. Bosson, D. Golshayan et al., "The diamant alpin dialysis cohort study: clinico-biological characteristics and cardiovascular genetic risk profile of incident patients," Journal of Nephrology, vol. 17, no. 1, pp. 66-75, 2004.

[47] A. N. N. Mertens and P. Holvoet, "Oxidized LDL and HDL: antagonists in atherothrombosis," FASEB Journal, vol. 15, no. 12, pp. 2073-2084, 2001.

[48] A. Brouwers, M. Langlois, J. Delanghe et al., "Oxidized lowdensity lipoprotein, iron stores, and haptoglobin polymorphism," Atherosclerosis, vol. 176, no. 1, pp. 189-195, 2004.

[49] A. P. Levy, J. E. Levy, S. Kalet-Litman et al., "Haptoglobin genotype is a determinant of iron, lipid peroxidation, and macrophage accumulation in the atherosclerotic plaque," Arteriosclerosis, Thrombosis, and Vascular Biology, vol. 27, no. 1, pp. 134-140, 2007.

[50] C. J. Roche, D. Dantsker, A. I. Alayash, and J. M. Friedman, "Enhanced nitrite reductase activity associated with the haptoglobin complexed hemoglobin dimer: functional and antioxidative implications," Nitric Oxide, vol. 27, no. 1, pp. 32-39, 2012.

[51] J. T. Sertorio, R. Lacchini, L. M. Amaral et al., "Haptoglobin polymorphism affects nitric oxide bioavailability in preeclampsia," Journal of Human Hypertension, 2012. 


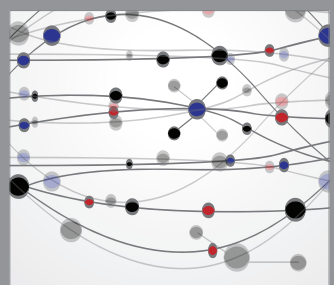

The Scientific World Journal
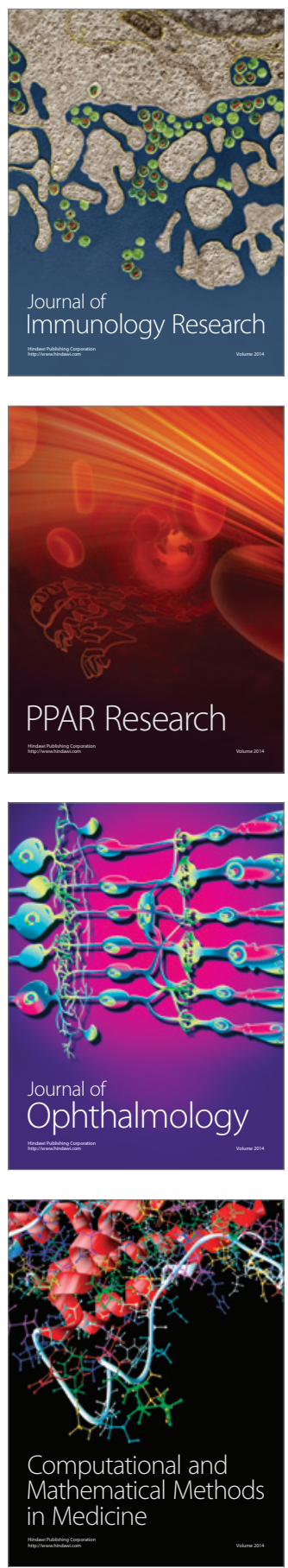

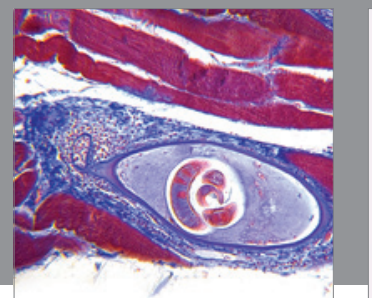

Gastroenterology

Research and Practice
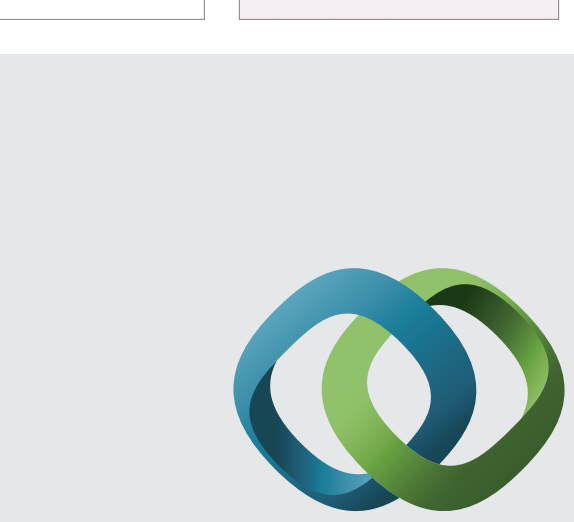

\section{Hindawi}

Submit your manuscripts at

http://www.hindawi.com
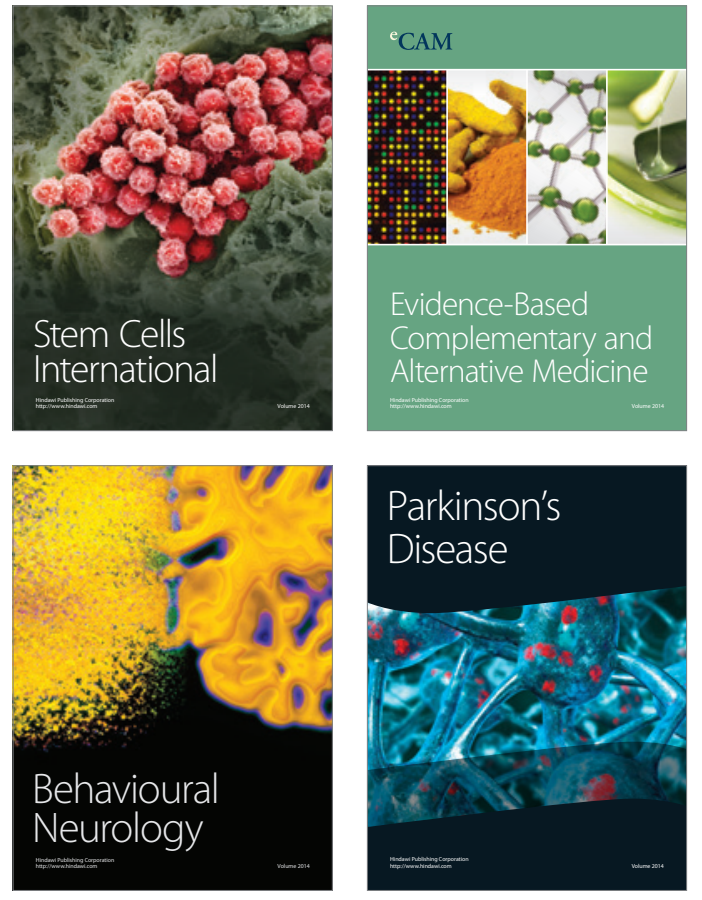
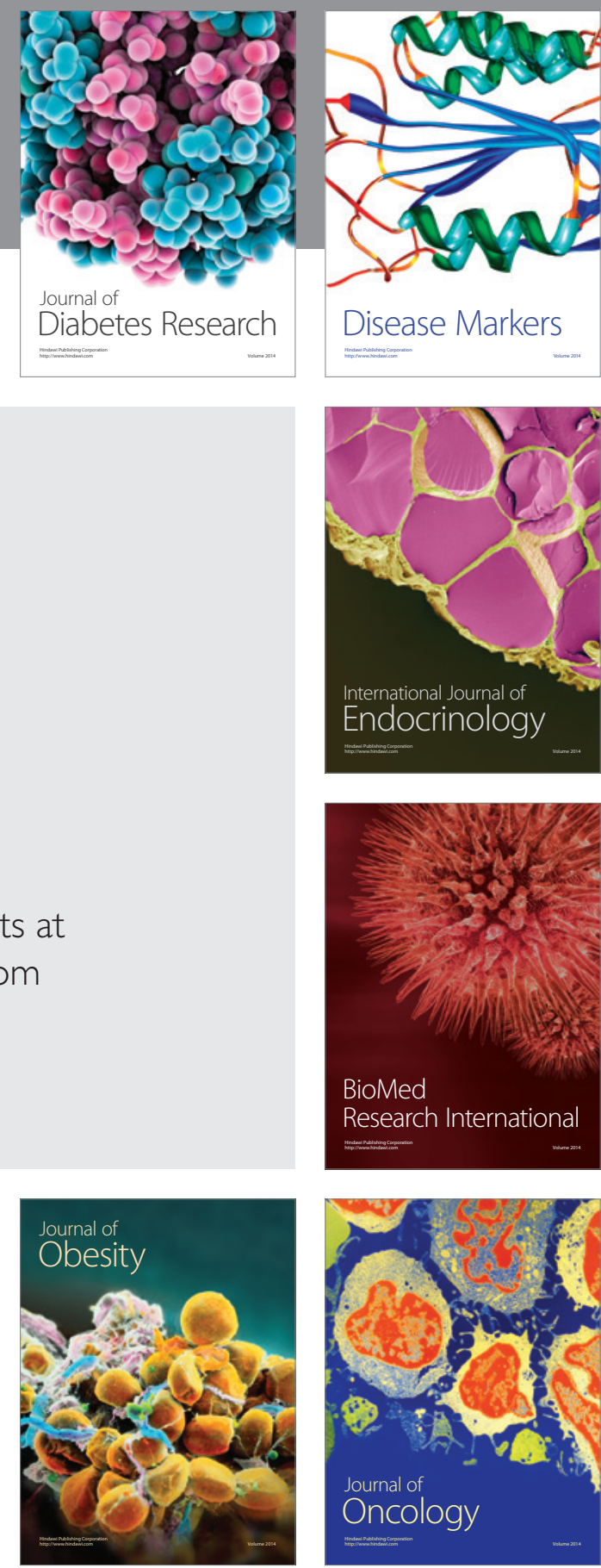

Disease Markers
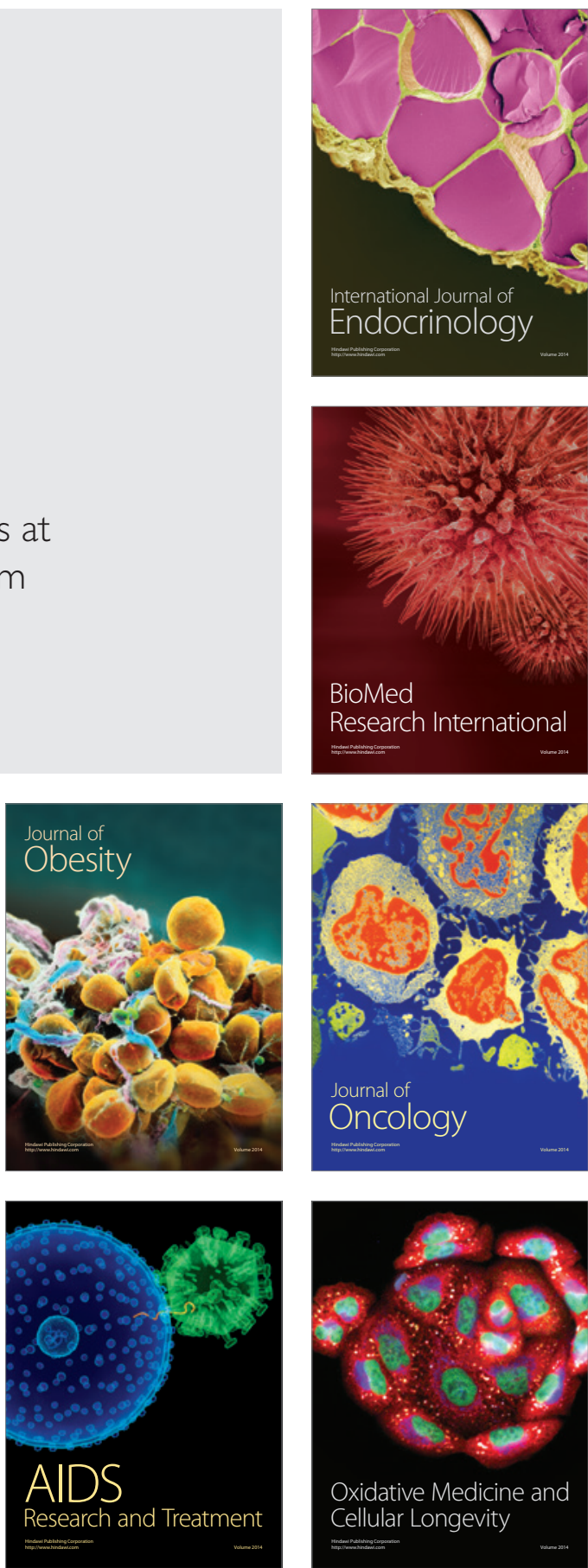